\title{
Personalidade e Câncer de Mama: Produção Científica em Psico-Oncologia ${ }^{1}$
}

\author{
Rodrigo Sanches Peres ${ }^{2}$ \\ Universidade Federal de Uberlândia \\ Manoel Antônio dos Santos \\ Universidade de São Paulo, Ribeirão Preto
}

\begin{abstract}
RESUMO - O presente estudo se insere no campo da Psico-Oncologia. Objetivou-se empreender uma revisão sistemática da literatura científica dedicada especificamente ao papel da personalidade na evolução da condição clínica e emocional de mulheres acometidas por câncer de mama. Foram consultadas as bases de dados MedLine, PsycINFO, LILACS, SciELOBrasil e PePSIC. De modo geral, os achados das referências selecionadas apontam que o otimismo enseja uma evolução mais favorável da condição emocional. Além disso, sustentam que pacientes com espírito de luta e manejo apropriado da ansiedade desencadeada por estímulos agressivos tendem a uma melhor condição clínica. Todavia, sugere-se a utilização, em futuras investigações, de escolhas teórico-metodológicas inovadoras para o avanço do conhecimento que atualmente se tem a respeito do assunto em pauta.
\end{abstract}

Palavras-chave: Psico-Oncologia; câncer de mama; personalidade; revisão da literatura.

\section{Personality and Breast Cancer: Scientific Production in Psycho-Oncology}

\begin{abstract}
This study concerns on the Psycho-Oncology field. It aimed to make a systematic review of the scientific literature about the personality importance in the development of emotional and clinical condition of women with breast cancer. This review was carried out on reports from MedLine, PsycINFO, LILACS, SciELO-Brasil and PePSIC databases. In general, the selected references' results point out that optimism promotes a more favorable evolution of emotional condition. Besides, they sustain that patients with fighting spirit and appropriate handling of anxiety evoked by aggressive stimuli tend to show a better clinical condition. However, it is suggested that future investigations should select innovative theoretical and methodological approaches for the improvement of the knowledge produced in the area.
\end{abstract}

Keywords: Psycho-Oncology; breast cancer; personality; literature review.

O câncer de mama é o tipo de neoplasia mais frequente em mulheres e se destaca como a terceira causa de morte entre brasileiras adultas. Recentes projeções do Ministério da Saúde apontam que aproximadamente 50.000 novos diagnósticos serão registrados somente em 2010 no território nacional e que o risco variará de 65 casos para cada 100.000 mulheres na região Sul a 30 casos para cada 100.000 mulheres na região Nordeste (Instituto Nacional do Câncer, 2009). As taxas de incidência aumentam anualmente, sobretudo como reflexo da tendência global à predominância de estilos de vida que fomentam a exposição a fatores de risco. Por essa razão, o controle do câncer de mama se destaca como uma preocupação crescente para os serviços nacionais de saúde pública.

Diversos autores, dentre os quais Massie e Holland (1991), Wanderley (1994), Rossi e Santos (2003), Venâncio (2004), Müller, Hoffmann e Fleck (2006) e Peres e Santos

1 O presente estudo é derivado de um projeto mais amplo, em nível de Doutorado, parcialmente subsidiado pela Coordenação de Aperfeiçoamento de Pessoal de Nível Superior (CAPES)

2 Endereço para corrrespondência: Universidade Federal de Uberlândia, Instituto de Psicologia. Avenida Pará, 1720 - Bloco 2C. Campus Umuarama. Uberlândia, MG. CEP 38401-136. Fone: (34) 98166401. E-mail: rodrigosanchesperes@yahoo.com.br.
(2007), afirmam que o câncer de mama incide sobre o principal símbolo corpóreo da feminilidade, da sensualidade, da sexualidade e da maternidade, de modo que compromete não somente a condição física da paciente, mas também sua saúde mental. Assim sendo, seu diagnóstico e tratamento representam um importante trauma na maioria dos casos, já que, além de afetarem as relações que a portadora mantém com seu corpo e com seu psiquismo, implicam na elaboração do luto decorrente da perda da vida "normal" anterior à enfermidade e na redefinição de planos, metas, sonhos e projetos futuros.

$O$ interesse da comunidade científica pelos aspectos psicológicos do câncer de mama aumentou consideravelmente nas duas últimas décadas, pois inúmeras pesquisas foram desenvolvidas desde os anos 1980 com esse enfoque. A maior parte delas tem como tema o impacto emocional do diagnóstico e do tratamento. A assistência psicológica a mulheres portadoras da doença igualmente se destaca como um assunto recorrente na literatura especializada. Ademais, os fatores psicológicos associados à gênese da enfermidade em questão também foram explorados por diversos estudos. Todavia, novas pesquisas se fazem necessárias para que possam ser esclarecidas certas questões para as quais, no atual estágio do conhecimento, ainda não existem respostas 
satisfatórias. Dentre essas questões é possível destacar a seguinte: de que forma o curso do câncer de mama pode ser influenciado pela personalidade da paciente?

Pesquisas que se propõem a abordar essa questão são potencialmente controversas, sobretudo porque o termo "personalidade" usualmente é empregado com uma multiplicidade de significados possíveis. Autores especializados - psicólogos ou psiquiatras como Fadiman e Frager (1979/1986), Lazarus e Monat (1979/1984), Ramos Júnior (1991), Dalgalarrondo (2000), D’Andrea (2000) e Schultz e Schultz (1994/2002) - apontam que o termo em questão pode ser entendido basicamente como a unidade individual resultante da interação de fatores biológicos, psicológicos, sociais e culturais que determina os sentimentos, os pensamentos e os comportamentos de um sujeito. Não obstante, certas concepções apregoam que a personalidade se transforma em resposta às situações às quais o indivíduo é exposto e outras, em contrapartida, defendem a existência de um conjunto mais estável de características pessoais.

Em face dessas considerações, evidencia-se a necessidade de demarcar os conhecimentos já estabelecidos e refletir sobre os desafios atuais e as novas perspectivas de pesquisas que se abrem nesse campo. Nessa vertente, o presente estudo objetivou empreender uma revisão sistemática da literatura dedicada especificamente ao papel da personalidade na evolução da condição clínica e emocional de mulheres acometidas por câncer de mama. As revisões sistemáticas da literatura científica, vale destacar, possuem um inegável valor prático, visto que facilitam o acesso aos resultados obtidos por um expressivo conjunto de pesquisadores relativos a um determinado tema e, consequentemente, permitem a identificação tanto de lacunas cujo preenchimento se faz necessário, quanto de repetições que devem ser evitadas no desenvolvimento de novas investigações (Alves-Mazzotti \& Gewandsnadjer, 2000).

\section{Método}

\section{Estratégias de busca das referências}

O material necessário para a revisão sistemática da literatura científica foi obtido mediante a execução de buscas eletrônicas em diferentes bases de dados on-line. Optou-se por adotar tal estratégia metodológica tendo-se em vista que a divulgação científica via internet, por ser mais ágil e menos onerosa, tem sido cada vez mais valorizada nos dias de hoje. Contudo, certas limitações são inerentes a qualquer uma das bases de dados existentes na atualidade. A combinação de diversas bases de dados, portanto, pode ser considerada a alternativa mais eficiente para que se possa executar um levantamento bibliográfico completo.

Partindo desse princípio, foram consultadas cinco bases de dados distintas, a saber: MedLine, PsycINFO, LILACS, SciELO-Brasil e PePSIC. As duas primeiras são mantidas, respectivamente, pela United States National Library of Medicine (NLM) e pela American Psychological Association (APA). Já as três últimas têm como órgãos responsáveis, respectivamente, o Centro Latino-Americano e do Caribe de
Informações em Ciências da Saúde (BIREME), a Fundação de Amparo à Pesquisa do Estado de São Paulo (FAPESP) e a Associação Brasileira de Editores Científicos de Psicologia (ABECiP).

Dentre as referidas bases de dados, duas - PsycINFO e PePSIC - são voltadas exclusivamente à divulgação de pesquisas científicas em Psicologia. Outras duas - MedLine e LILACS - são especializadas em Ciências da Saúde. A SciELO-Brasil, por sua vez, é multidisciplinar. Todas elas, contudo, têm em comum o fato de serem dirigidas por instituições reconhecidamente comprometidas com o avanço e a difusão do conhecimento. Ressalte-se ainda que, tomadas em seu conjunto, as referidas bases de dados possibilitam a realização de buscas em mais de 10.000 periódicos científicos originários de dezenas de países, de modo que são instrumentos capazes de subsidiar a execução de um levantamento bibliográfico merecedor de crédito e representativo do estado da arte.

As buscas eletrônicas foram executadas a partir da utilização dos descritores "personalidade" (personality) e "neoplasias mamárias" (breast neoplasms) e do operador booleano "e" (and). Tal estratégia foi privilegiada levando-se em consideração que viabiliza a localização de referências que apresentam os dois descritores adotados. As bases de dados foram configuradas para localizar as referências que apresentavam o descritor "neoplasias mamárias" entre as palavras-chave e o descritor "personalidade" no resumo. Esse procedimento foi adotado para possibilitar a obtenção de um resultado mais preciso do que aquele que poderia ser atingido sem a especificação dos campos de busca.

Optou-se pelo emprego dos referidos descritores levandose em conta que ambos são reconhecidos pelas bases de dados em questão e utilizados de forma corrente na literatura científica especializada. Ressalte-se que o descritor "neoplasias mamárias" foi selecionado porque ocupa o topo de uma das classes constituintes da estrutura hierárquica que organiza os principais índices de termos técnicos no campo da saúde, tais como o "Medical Subject Headings" e o "Descritores em Ciências da Saúde". Desse modo, o procedimento em questão viabiliza a execução de buscas mais amplas, na medida em que permite a localização de referências que adotam quaisquer descritores da mesma classe, sendo um deles o descritor "câncer de mama". Cumpre assinalar ainda que, para subsidiar um levantamento bibliográfico atualizado e abrangente, as bases de dados foram configuradas para localizar as referências publicadas entre 1995 e 2005.

\section{Procedimentos para seleção e apreciação das referências}

O título e a autoria de todas as referências obtidas a partir das buscas eletrônicas foram inicialmente examinados visando a subsidiar a eliminação de eventuais repetições. A seguir, os resumos das referências selecionadas mediante o emprego desse procedimento foram submetidos a uma leitura preliminar, cujo propósito básico foi determinar o material efetivamente pertinente a essa revisão sistemática da literatura. Tal leitura foi norteada em função de critérios de inclusão específicos, que permitiram qualificar os traba- 
lhos. O primeiro deles é o idioma. Foram consideradas as referências escritas em língua inglesa, francesa, espanhola ou portuguesa.

O segundo critério de inclusão é o formato. Apenas as referências publicadas como artigo, relato de experiência ou revisão da literatura científica foram selecionadas. Consequentemente, foram descartadas as referências publicadas como livro, capítulo de livro, dissertação, tese, resenha, resumo ou carta ao editor. O terceiro critério, por fim, é o objetivo. Foram consideradas somente as referências voltadas especificamente ao estudo da influência da personalidade no curso da condição clínica e emocional de mulheres acometidas por câncer de mama. Assim sendo, foram preteridas as referências relacionadas a outros aspectos psicológicos da doença em questão.

As referências selecionadas foram recuperadas na íntegra e submetidas a uma apreciação qualitativa com o auxílio desses critérios e mediante a execução de leituras analíticas de cada uma delas. Para melhor organização, os achados oriundos dessas leituras foram analisados a partir da identificação de 10 dimensões de análise, a saber: (1) modalidade de produção científica; (2) autoria e filiação institucional; (3) fonte; (4) tema específico; (5) amostra; (6) abordagem metodológica; (7) delineamento de pesquisa; (8) fundamentação teórica; (9) instrumentos; e (10) principais resultados. A opção por tais dimensões de análise, vale destacar, levou em consideração os procedimentos originalmente propostos em uma revisão sistemática da literatura anterior (Peres \& Santos, 2006).

\section{Resultados e Discussão}

\section{Apreciação quantitativa das referências}

As buscas eletrônicas possibilitaram a localização de 135 referências, sendo 69 delas na PsycINFO e 66 na MedLine. Nas demais bases de dados consultadas - LILACS, SciELO-Brasil e PePSIC - não foi encontrada sequer uma referência, o que evidencia que, de 1995 a 2005, nenhum estudo dedicado especificamente à influência da personalidade no curso da condição clínica e emocional de mulheres acometidas por câncer de mama foi publicado em periódicos científicos latino-americanos indexados nas bases selecionadas. Desse modo, constata-se de antemão uma lacuna significativa a ser preenchida com o conhecimento oriundo de novas pesquisas.

A checagem do título e da autoria das referências inicialmente obtidas apontou que 26 trabalhos foram localizados tanto na PsycINFO quanto na MedLine. O número de referências foi reduzido, portanto, de 135 para 109, pois as repetições foram automaticamente descartadas. A seguir, os resumos das referências selecionadas com esse primeiro procedimento foram submetidos a uma leitura preliminar, conduzida a partir da utilização dos critérios de inclusão estabelecidos. Essa leitura implicou, no total, na eliminação de mais 90 referências. Assim sendo, foram efetivamente consideradas pertinentes a essa revisão da literatura apenas 19 das 135 referências inicialmente obtidas. O título de cada uma dessas 19 referências, na ordem em que as mesmas foram localizadas, respectivamente, na PsycINFO e na MedLine, encontra-se reproduzido na Tabela 1.

A discrepância verificada entre o número de referências inicialmente obtido mediante a busca nos sistemas eletrônicos de identificação bibliográfica empregados e a quantidade de referências considerada efetivamente pertinente já era esperada em virtude do estabelecimento de critérios de inclusão rigorosos. Cumpre assinalar, contudo, que tais critérios se mostraram de extrema importância, pois permitiram selecionar adequadamente as referências inicialmente obtidas, conferindo, consequentemente, maior precisão a esta revisão da literatura. Isso se torna especialmente claro quando se observa a quantidade de referências excluídas por não atenderem ao terceiro critério de inclusão estabelecido.

\section{Apreciação qualitativa das referências}

A avaliação da primeira dimensão de análise (modalidade de produção científica) indica que todas as referências selecionadas apresentam dados empíricos oriundos de trabalhos originais. A princípio, poder-se-ia considerar positivo esse achado. Afinal, o número reduzido de pesquisas dedicadas especificamente à influência da personalidade no curso da condição clínica e emocional de mulheres acometidas por câncer de mama poderia sugerir que o assunto ainda não foi exaustivamente explorado. Em contrapartida, a inexistência de revisões bibliográficas pode ser considerada desfavorável, pois, como já mencionado, trabalhos desse tipo possibilitam a obtenção de um panorama abrangente da produção científica e fornecem valiosas indicações para novas pesquisas, de modo que possuem um inegável valor prático.

Charles S. Carver e Michael H. Antoni, ambos psicólogos e docentes do Center for Psycho-Oncology Research da University of Miami, destacaram-se pelos resultados da avaliação da segunda dimensão de análise (autoria), visto que assinam três referências (Carver, Meyer \& Antoni, 2000; Carver \& cols., 2005; Urcuyo, Boyers, Carver \& Antoni, 2005) sobre o tema em questão. Ressalte-se, ainda, que os noruegueses Lars Tjemsland, Jon A. Soreide e Ulrik F. Malt, médicos do Rogaland Central Hospital e do Norway National Hospital, constam entre os autores de dois estudos (Tjemsland, Soreide \& Malt, 1998; Tjemsland, Soreide, Matre \& Malt, 1997). Além disso, trabalhando com grupos de pesquisa distintos, Yosuke Uchitomi, psiquiatra da Psycho-Oncology Division do National Cancer Research Institute East, no Japão, também assina dois estudos (Okamura, Yamawaki, Akechi, Taniguchi \& Uchitomi, 2005; Sugawara \& cols., 2005).

A avaliação da terceira dimensão de análise (fonte) aponta que grande parte das referências selecionadas (Bleiker, Pouwer, Van der Ploeg, Leer \& Ader, 2000; Fredette, 1995; Golden-Kreutz \& Andersen, 2004; Millar, Purushotham, McLatchie, George \& Murray, 2005; Rhodes, Courneya \& Bobick, 2001; Schou, Ekeberg, Sandvik, Hjermstad \& Ruland, 2005; Sugawara \& cols., 2005; Tjemsland \& cols., 1997) foi publicada em periódicos de natureza multidisciplinar, dentre os quais o Journal of Psychossomatic Research e o Supportive Care in Cancer. Todavia, periódicos com ênfase psicológica, tais como o Health Psychology e o Journal of 
Tabela 1. Ordem e título das referências selecionadas.

\begin{tabular}{|c|c|}
\hline Ordem & Título \\
\hline A & $\begin{array}{l}\text { Multiple predictors of health-related quality of life in early stage breast cancer: Data from a year follow-up study compared } \\
\text { with the general population }\end{array}$ \\
\hline $\mathrm{B}$ & $\begin{array}{l}\text { Optimistic personality and psychosocial well-being during treatment predict psychosocial well-being among long-term } \\
\text { survivors of breast cancer }\end{array}$ \\
\hline $\mathrm{C}$ & A 1-year prospective study of individual variation in distress, and illness perceptions, after treatment for breast cancer \\
\hline $\mathrm{D}$ & Finding benefit in breast cancer: Relations with personality, coping, and concurrent well-being \\
\hline $\mathrm{E}$ & Depressive symptoms after breast cancer surgery: Relationships with global, cancer-related, and life event stress \\
\hline $\mathrm{F}$ & Locus of control, marital status and predictors of early relapse in primary breast cancer patients \\
\hline G & Personality and exercise participation across the breast cancer experience \\
\hline $\mathrm{H}$ & The relationship between agentic and communal personality traits and psychosocial adjustment to breast cancer \\
\hline I & $\begin{array}{l}\text { Responsiveness to threats and incentives, expectancy of recurrence, and distress and disengagement: Moderator effects in } \\
\text { women with early stage breast cancer }\end{array}$ \\
\hline $\mathrm{J}$ & Breast cancer, coping and quality of life: A semi-prospective study \\
\hline $\mathrm{K}$ & Psychological distress two years after diagnosis of breast cancer: Frequency and prediction \\
\hline $\mathrm{L}$ & Properative psychological variables predict immunological status in patients with operable breast cancer \\
\hline M & $\begin{array}{l}\text { Psychiatric disorders following first breast cancer recurrence: Prevalence, associated factors and relationship to quality of } \\
\text { life }\end{array}$ \\
\hline $\mathrm{N}$ & Predictors of quality of life in newly diagnosed melanoma and breast cancer patients \\
\hline $\mathrm{O}$ & Occurrence of fatigue and associated factors in disease-free breast cancer patients without depression \\
\hline $\mathrm{P}$ & $\begin{array}{l}\text { Psychological profile in patients with stages I and II breast cancer: Associations of psychological profile with tumor biolo- } \\
\text { gical prognosticators }\end{array}$ \\
\hline Q & Psychological factors can predict the response to primary chemotherapy in patients with locally advanced breast cancer \\
\hline $\mathrm{R}$ & Posttraumatic distress symptoms in operable breast cancer III: Status one year after surgery \\
\hline S & Breast cancer survivors: Concerns and coping \\
\hline
\end{tabular}

Consulting and Clinical Psychology, também veicularam uma parcela significativa das referências (Carver \& cols., 2000; Carver \& cols., 2005; Cousson-Gelie, 2000; Declerck, De Brabander, Boone \& Gerits, 2002; Lilja \& cols., 2003; Piro, Zeldow, Knight, Mytko \& Gradishar, 2001; Urcuyo \& cols., 2005). Deve-se salientar também que, embora tenha se revelado discreta nessa revisão da literatura, a penetração de estudos voltados aos mais diversos aspectos emocionais do câncer em periódicos com ênfase médica tem, conforme se deduz a partir de um levantamento recente executado por Levin e Kissane (2006), apresentado-se como uma tendência promissora.

Ainda a propósito da avaliação dessa dimensão de análise, ressalte-se que o periódico Psycho-Oncology, publicado em parceria pela International Psycho-Oncology Society, pela American Psychosocial Oncology Society e pela British Psycho-Oncology Society, sobressai-se como o principal veículo de divulgação das referências selecionadas. A opção dos autores por esse periódico pode ser considerada oportuna, partindo-se do princípio de que o termo que lhe dá nome Psico-Oncologia - diz respeito, segundo a clássica definição de Holland (1990), à área de interface entre a Psicologia e a Oncologia. Essa área se dedica, segundo a referida autora, ao estudo de duas dimensões psicológicas básicas do câncer, a saber: o impacto da doença na condição emocional do paciente, de seus familiares e dos profissionais de saúde envolvidos em seu tratamento e o papel de variáveis psicológicas na incidência e na sobrevivência à doença.

A avaliação da quarta dimensão de análise (temas específicos) demonstra que, a despeito de tratarem de um assunto em comum, as referências selecionadas se caracterizam por certa diversidade. Uma parcela significativa delas (Bleiker \& cols., 2000; Carver \& cols., 2000; Carver \& cols., 2005; Fredette, 1995; Golden-Kreutz \& Andersen, 2004; Lehto, Ojanen \& Kellokumpu-Lehtinen, 2005; Piro \& cols., 2001; Schou \& cols., 2005; Urcuyo \& cols., 2005) é dedicada à investigação de relações entre certas facetas da personalidade e determinados indicadores da condição emocional de mulheres acometidas pelo câncer de mama. A opção dos autores por variáveis independentes mais circunscritas é um recurso necessário diante das dificuldades metodológicas inerentes à avaliação da personalidade, as quais decorrem da abran- 
gência do construto em questão. Porém, como alerta Hogan (2003/2006), a medida de um traço psicológico isolado não pode ser considerada representativa da personalidade como um todo.

O enfrentamento - termo correspondente em língua portuguesa ao verbete inglês coping - é o traço psicológico de avaliação mais usual entre as referências selecionadas, visto que foi tomado como variável independente, principal ou secundária, em seis delas (Cousson-Gelie, 2000; Fredette, 1995; Lehto \& cols., 2005; Lilja \& cols., 2003; Tjemsland \& cols., 1997; Urcuyo \& cols., 2005). Tal construto pode ser definido basicamente como o conjunto de operações executadas por uma pessoa com o intuito de se adaptar às circunstâncias adversas (Antoniazzi, Dell'Aglio \& Bandeira, 1998). Originalmente, o enfrentamento foi concebido pela psicologia do ego como um correlato dos mecanismos de defesa adotados inconscientemente com o propósito de proteger o aparelho psíquico de uma eventual desestruturação. Desse modo, seria totalmente determinado pela personalidade do indivíduo e pouco se alteraria de uma situação para outra (Hartmann, 1958).

Em um segundo momento, o enfrentamento foi redefinido a partir de uma perspectiva cognitivista como o conjunto de esforços deliberadamente empreendidos por um sujeito para minimizar a qualidade aversiva de um evento estressor e, assim, criar, aumentar ou ampliar a sensação de controle sobre o mesmo (Lazarus \& Folkman, 1984). Tratar-se-ia, portanto, de um processo direcionado por transações com o ambiente. Ou seja: seria aprendido e poderia ser aprimorado. Todavia, a noção de avaliação do evento estressor é central nessa perspectiva, pois a forma como o mesmo é percebido por meio de processos cognitivos individuais também influenciaria o enfrentamento, potencializando ou restringindo recursos internos.

Recentemente, uma proposta teórica conciliadora tem se voltado mais uma vez ao estudo das convergências entre a personalidade e o enfrentamento (Antoniazzi \& cols., 1998). De acordo com tal proposta, porém, é preciso distinguir dois construtos anteriormente empregados como sinônimos: estilos de enfrentamento e estratégias de enfrentamento. $\mathrm{O}$ primeiro se refere, conforme Carver e Scheier (1994), a uma predisposição a responder de maneira habitual a diferentes eventos estressores e decorre de características mais estáveis da personalidade. Já o segundo diz respeito a ações bastante variáveis, de determinação essencialmente situacional, voltadas à mediação entre um dado evento estressor e suas consequências (Folkman \& cols., 1986).

Os estilos de enfrentamento foram preteridos em prol das estratégias de enfrentamento no contexto das referências selecionadas, o que, tendo em vista a definição de cada um desses construtos, limita, em certo grau, o alcance dos resultados obtidos pelos autores no que tange especificamente à abrangência da compreensão da personalidade das pacientes acometidas por câncer de mama que compuseram suas amostras. Em contrapartida, a escolha das variáveis dependentes parece ter sido mais apropriada, pois foram tomados como parâmetros da condição física e emocional das mulheres avaliadas basicamente construtos que articulam domínios de naturezas distintas, a saber: qualidade de vida e estresse.
Contemplada em cinco referências (Carver \& cols., 2005; Cousson-Gelie, 2000; Lehto \& cols., 2005; Okamura \& cols., 2005; Schou \& cols., 2005), a qualidade de vida surge como noção pouco precisa nos anos 70 do século passado em consequência do movimento de humanização na assistência em saúde, o qual salientava a necessidade de uma maior valorização de critérios centrados nos pacientes para a avaliação da resolutividade de tratamentos médicos. Seu emprego rapidamente se ampliou, tanto no meio científico quanto na população em geral. Porém, somente duas décadas depois a qualidade de vida foi operacionalmente estruturada pela Organização Mundial de Saúde em torno de seis facetas complementares: aspectos físicos, nível de independência, relações pessoais, aspectos psicológicos, meio ambiente e espiritualidade (World Health Organization Quality of Life Group, 1994).

A partir dessa estruturação, a qualidade de vida ascendeu ao estatuto de construto e passou a ser definida, em uma perspectiva transcultural, como a percepção do indivíduo de sua posição na vida, no contexto do sistema de valores nos quais ele se encontra inserido e em relação aos seus objetivos, expectativas, padrões e preocupações (World Health Organization Quality of Life Group, 1994). Essa definição, não obstante, ainda é alvo de questionamentos. Por essa razão, certos autores, enfatizando o papel do estado de saúde - seja ele subjetivo ou objetivo - no funcionamento cotidiano em termos emocionais, sociais e produtivos, preferem o emprego de conceitos derivados, tais como qualidade de vida relacionada à saúde (Seidl \& Zannon, 2004).

Cumpre assinalar também que o estresse, variável dependente em cinco referências (Bleiker \& cols., 2000; Carver \& cols., 2000; Declerck \& cols., 2002; Millar \& cols., 2005; Tjemsland \& cols., 1998), é definido de modo mais pontual. Ainda assim, agrega os domínios físico e emocional, já que é utilizado classicamente para fazer menção às reações psicológicas e orgânicas apresentadas por um indivíduo quando submetido a circunstâncias que abalam sua homeostase (Selye, 1965). Essas reações podem impulsionar seu desempenho e favorecer seu ajustamento, trazendo, como consequência, efeitos positivos. Quando isso acontece, caracteriza-se o quadro de eutress. Por outro lado, essas reações podem minimizar a eficácia de seus recursos adaptativos e conduzir a um desgaste crônico. Nesses casos, instala-se o quadro de distress (Sparremberger, Santos \& Lima, 2003). A identificação do quadro de distress é visada de modo direto em quatro das cinco referências mencionadas (Carver \& cols., 2000; Declerck \& cols., 2002; Millar \& cols., 2005; Tjemsland \& cols., 1998).

A avaliação da quinta dimensão de análise (amostra) indica que, de modo geral, os autores das referências selecionadas optaram por amostras não-probabilísticas de conveniência, uma vez que incluíram mulheres acometidas por câncer de mama que apresentavam duas características básicas: (a) foram consecutivamente admitidas para tratamento ou reabilitação em instituições de saúde durante um dado período; e (b) preenchiam requisitos clínicos ou sociodemográficos básicos previamente sistematizados. Indubitavelmente se encontra associado a esse fato o expressivo número de participantes da maioria das referências selecionadas, situado entre 100 e 200 em sete delas (Bleiker \& cols., 2000; Carver \& cols., 2005; 
Lehto \& cols., 2005; Lilja \& cols., 2003; Rhodes \& cols., 2001; Schou \& cols., 2005; Tjemsland \& cols., 1997).

A predileção pela abordagem quantitativa no contexto das referências selecionadas se torna patente com a avaliação da sexta dimensão de análise (abordagem metodológica). Afinal, em apenas duas delas (Declerck \& cols., 2002; Fredette, 1995) a abordagem qualitativa foi adotada. É notório que indicações e contra-indicações são inerentes a ambas as abordagens, de modo que, como sustentam Minayo e Sanches (1993), confrontá-las com o intuito de identificar uma suposta hierarquia se afigura como um procedimento injustificável. Não obstante, é possível propor que o conhecimento produzido sobre o assunto do qual trata essa revisão da literatura se encontra demasiadamente atrelado ao paradigma positivista e à ontologia objetivista, o que, em última instância, sugere a equivocada desvalorização de incursões compreensivistas.

Conforme a avaliação da sétima dimensão de análise (delineamento de pesquisa), os autores de 12 referências selecionadas se apoiaram em um conjunto de medidas que, levando em conta o fator tempo, foram obtidas visando ao estabelecimento de eventuais relações entre certos efeitos e suas supostas condições associadas, o que caracteriza o delineamento longitudinal. A maioria delas (Bleiker \& cols., 2000; Carver \& cols., 2005; Cousson-Gelie, 2000; Declerck \& cols., 2002; Golden-Kreutz \& Andersen, 2004; Millar \& cols., 2005; Schou \& cols., 2005; Tjemsland \& cols., 1997; Walker \& cols., 1999) focalizava especificamente efeitos observáveis após o início da coleta de dados, de modo que os resultados dependeram da conservação da amostra. Conclui-se, portanto, que o delineamento longitudinal do tipo prospectivo foi o mais frequente. De acordo com Forattini (1996), estudos com esse caráter fornecem informações mais acuradas do que os estudos longitudinais retrospectivos ou os estudos transversais, o que autoriza a atribuição de um selo de qualidade metodológica às referências mencionadas.

No contexto das referências selecionadas, as teorias de orientação cognitivo-comportamental - tais como a teoria da adaptação cognitiva de Vicki Helgeson e a teoria da avaliação cognitiva de Susan Folkman e Richard S. Lazarus - têm clara primazia, posto que foram empregadas na maioria das referências selecionadas (Carver \& cols., 2005; Carver \& cols., 2000; Cousson-Gelie; 2000; Fredette, 1995; Lehto \& cols., 2005; Piro \& cols., 2001; Urcuyo \& cols., 2005). Somente uma referência (Lilja \& cols., 2003) se diferencia pela utilização de uma teoria psicodinâmica, de acordo com a avaliação da oitava dimensão de análise (fundamentação teórica). Pode-se atribuir a prevalência das teorias de orientação cognitivo-comportamental à popularização da medicina comportamental nas últimas décadas. Afinal, tal perspectiva teórica surgiu como resultado da necessidade de sistematizar o conhecimento produzido pelas ciências humanas e biomédicas sobre processos cognitivos conscientes e comportamentos observáveis de relevância no âmbito da saúde (Schwartz \& Weiss, 1978).

Diante do exposto, constata-se que a diversidade teórica que caracteriza a Psicologia não se reflete no estudo da influência da personalidade no curso da condição clínica e emocional de mulheres acometidas por câncer de mama. É possível cogitar que a reversão desse quadro teria desdobramentos potencialmente favoráveis para o avanço do conhecimento, dado que o assunto do qual trata a presente revisão da literatura não se encontra esgotado. Ademais, deve-se destacar que, para Holland (2002), a efetiva integração de aspectos objetivos e subjetivos das experiências vivenciadas pelos pacientes a partir do diagnóstico do câncer é imprescindível para a superação de barreiras ao desenvolvimento da PsicoOncologia como um todo.

A avaliação da nona dimensão de análise (instrumentos) testemunha que, na maioria das referências em que se admitiu de fato a personalidade como variável independente (Millar \& cols., 2005; Okamura \& cols., 2005; Sugawara \& cols., 2005; Tjemsland \& cols., 1998; Tjemsland \& cols., 1997; Walker e cols., 1999), o Eysenck Personality Questionnaire-Revised foi utilizado. Tal instrumento psicométrico parte do princípio de que a personalidade é composta por três combinações de fatores que se situam em extremos, a saber: extroversão/ introversão, neuroticismo/estabilidade emocional e psicoticismo/controle dos impulsos (Eysenck, 1990). Portanto, a chamada abordagem dos traços - modelo conceitual clássico que, segundo Schultz e Schultz (1994/2002), preconiza a estruturação da personalidade em torno de um conjunto determinado de características diferenciadoras que regem o comportamento - também se destaca como um marco teórico importante no contexto das referências selecionadas.

Por outro lado, a avaliação das estratégias de enfrentamento foi diversificada. Em três das seis referências em que esse construto se afigura como variável independente (Cousson-Gelie; 2000; Lehto \& cols., 2005; Urcuyo \& cols., 2005), instrumentos psicométricos de base cognitivocomportamental foram adotados: o Brief COPE Inventory, o Ways of Coping Checklist e o Ways of Coping Questionnaire, respectivamente. Nas outras três, os autores optaram por entrevistas psicológicas não-padronizadas (Fredette, 1995; Lilja \& cols., 2003; Tjemsland \& cols., 1997). Ainda sobre a avaliação das variáveis independentes, é preciso mencionar o emprego do Life Orientation Test-Revised em três referências (Carver \& cols., 2005; Schou \& cols., 2005; Urcuyo \& cols., 2005). Trata-se de um instrumento psicométrico voltado especificamente à mensuração da tendência individual ao otimismo.

Instrumentos psicométricos também foram privilegiados na avaliação das variáveis dependentes. No que se refere à qualidade de vida, os questionários elaborados pela European Organization for Research and Treatment of Cancer se destacaram como os mais frequentes, posto que foram utilizados em quatro referências (Cousson-Gelie, 2000; Lehto \& cols., 2005; Okamura \& cols., 2005; Schou \& cols., 2005). Já a mensuração do estresse foi efetuada indiretamente em outras quatro referências, mediante a combinação dos resultados obtidos a partir da aplicação de instrumentos voltados à identificação de sintomas de ansiedade e depressão ou à mensuração do funcionamento social e do impacto emocional da doença (Bleiker \& cols., 2000; Declerck \& cols., 2002; Millar \& cols., 2005; Tjemsland \& cols., 1998), tais como o General Health Questionnaire-Reduced Version, o Symptom Checklist, o Social Experience Checklist e a Impact of Events Scale.

A partir da avaliação da décima dimensão de análise (principais resultados), constata-se que parte das referências dedicadas ao estudo das relações entre a personalidade e o 
curso da condição emocional de mulheres acometidas por câncer de mama sustenta que o otimismo se afigura como uma espécie de proteção contra o impacto psicológico da doença (Carver \& cols., 2005; Millar \& cols., 2005; Schou \& cols., 2005). Afinal, pacientes que apresentam uma reação ao diagnóstico influenciada por essa característica são propensas, como ilustram os achados das referências em questão, a apreciar de forma positiva sua qualidade de vida, a veicular uma imagem corporal favorável, a referir um funcionamento sexual satisfatório e até mesmo a relatar benefícios emocionais e existenciais associados ao adoecimento, tanto durante o tratamento quanto após a remissão da enfermidade.

Resultados análogos são recorrentes em pesquisas desenvolvidas junto a portadores de diversos tipos de câncer (Scheier, Carver \& Bridges, 2001). Não obstante, deve-se mencionar que achados dessa natureza podem, quando levados ao extremo e generalizados sem cautela, conduzir a um fenômeno nocivo conhecido como "tirania do pensamento positivo". Tal fenômeno, descrito por Holland e Lewis (2000), faz com que o paciente oncológico se sinta pressionado - tanto interna quanto externamente - a experimentar apenas emoções consideradas positivas e, assim, a reprimir sentimentos supostamente negativos, como se disso dependesse sua sobrevivência. Dessa maneira, enseja uma postura estereotipada, a qual frequentemente evoluiu da resignação estóica à culpabilidade, uma vez que o menor revés no tratamento abala sua sustentação.

A utilização, durante o tratamento, de estratégias de enfrentamento centradas no problema - ou seja, capazes de subsidiar uma ação direta sobre a situação estressora (Lazarus \& Folkman, 1984) - favorece, de acordo com duas referências, uma auto-avaliação positiva da condição emocional em mulheres recém submetidas à cirurgia (Urcuyo \& cols., 2005) e a uma redefinição de valores e prioridades em pacientes já livres da doença (Fredette, 1995). Porém, um dos estudos (Cousson-Gelie, 2000) alerta que, quando associado à perda subjetiva de controle, o emprego de estratégias de enfrentamento dessa natureza pode promover o oposto. Isso possivelmente ocorre porque a imprevisibilidade que caracteriza a evolução da doença pode ceifar o desejo de resgatar simbolicamente as rédeas da própria vida e, como consequência, afetar o senso de autoeficácia e comprometer a autoestima em certos casos (Gimenes, 1997).

Cumpre assinalar que o neuroticismo é apontado em quatro referências (Golden-Kreutz \& Andersen, 2004; Millar \& cols., 2005; Okamura \& cols., 2005; Tjemsland \& cols., 1998) como uma característica de personalidade determinante para o distress em pacientes acometidas por câncer de mama, pois aumenta a suscetibilidade à tensão constante, nervosismo, expectativa apreensiva, infelicidade e anedonia. Assim, pode precipitar a eclosão de sintomas clinicamente significativos de ansiedade, depressão e transtorno de estresse pós-traumático. Outras duas referências relacionam o neuroticismo ao sedentarismo e à fadiga crônica até mesmo após a remissão da enfermidade (Rhodes \& cols., 2001; Sugawara \& cols., 2005), o que o credencia como preditor de prejuízos do reajustamento funcional nessa população, dado que a prática de exercícios físicos notoriamente previne complicações e minimiza limitações decorrentes do tratamento, além de favorecer o bem-estar psicológico.
Ademais, segundo o conjunto de referências selecionadas nessa revisão da literatura, também podem ser consideradas em situação de risco em termos emocionais mulheres que, antes do diagnóstico, vivenciaram eventos estressores (Golden-Kreutz \& Andersen, 2004) e, ao longo do tratamento, referem crenças errôneas sobre o câncer de mama (Millar \& cols., 2005), apresentam um padrão de expressão emocional caracterizado pela inibição e um padrão de relacionamento interpessoal marcado pela negligência de suas próprias necessidades (Piro \& cols., 2001), demonstram auto-acusação (Cousson-Gelie, 2000), comportamento de evitação relacionado à doença (Bleiker \& cols., 2000), elevada responsividade a ameaças e reduzida responsividade a recompensas (Carver \& cols., 2000), situação de comorbidade psiquiátrica (Okamura \& cols., 2005), ou ainda que adotam estratégias de enfrentamento centradas na emoção (Lehto \& cols., 2005) e avaliam de forma negativa o suporte social recebido (Tjemsland \& cols., 1998).

No que tange à influência da personalidade no curso da condição clínica de mulheres acometidas por câncer de mama, é preciso mencionar que, de acordo com os achados de duas investigações selecionadas, pacientes que sofrem um comprometimento clinicamente significativo da condição emocional quando da descoberta da enfermidade tendem a apresentar níveis reduzidos de linfócitos B e células T4 após a cirurgia (Tjemsland \& cols., 1997) e a obter resultados pouco efetivos com a quimioterapia (Walker \& cols., 1999). Na esteira desses achados, outra referência sustenta que mulheres que demonstram locus de controle intermediário, referem satisfação com o suporte familiar e não experimentam perda subjetiva de controle são pouco propensas à recidiva precoce (Declerck \& cols., 2002).

Por fim, uma referência indica que pacientes que, a despeito de inicialmente reagirem com desesperança ao diagnóstico, adotam estratégias de enfrentamento centradas no problema (principalmente espírito de luta) e se mostram capazes de manejar satisfatoriamente a ansiedade provocada por estímulos agressivos, além de serem propensas a regressões a serviço do ego, tendem a apresentar tumores com indicadores prognósticos anatômicos, histológicos e endócrinos favoráveis, dentre os quais extensão inferior a três centímetros, baixo índice proliferativo, linfonodos axilares preservados e receptor hormonal positivo (Lilja \& cols., 2003). Além de contribuírem para um maior período de sobrevida e um menor risco de recorrência da doença, tais indicadores justificam procedimentos cirúrgicos conservadores e contra-indicam terapêuticas adjuvantes (Abreu \& Koifman, 2002).

\section{Considerações Finais}

A presente revisão da literatura permitiu traçar um rico e abrangente panorama dos estudos em âmbito internacional que se dedicaram a investigar as relações entre o curso do câncer de mama e a personalidade de pacientes acometidas pela doença. Além disso, demarcou limites e apontou potencialidades encontradas nesse campo do conhecimento, a partir de evidências oriundas de referências confiáveis que podem estimular o interesse dos pesquisadores brasileiros 
por essa temática. Para tanto, foram discutidas questões conceituais a respeito dos construtos colocados em relevo pelos estudos, que posssibilitam o delineamento dos desafios atuais e novas perspectivas para a produção científica em Psico-Oncologia.

A compreensão pormenorizada da influência da personalidade na evolução da condição clínica e emocional de mulheres acometidas por câncer de mama indubitavelmente depende de novos estudos. Porém, deve-se destacar que a execução de escolhas teórico-metodológicas inovadoras em relação àquelas que foram privilegiadas pelos autores das referências selecionadas na presente revisão da literatura pode ser considerada um caminho promissor para o avanço do conhecimento que, atualmente, se tem a respeito do assunto. Assim, sugere-se o emprego, em futuras investigações, de abordagens qualitativas e da adoção de teorias capazes de contemplar os aspectos mais profundos das vivências emocionais apresentadas por essa população antes, durante e após o adoecimento.

Ressalte-se ainda que, devido à hegemonia dos instrumentos psicométricos - e também das teorias de orientação cognitivo-comportamental - no contexto das referências selecionadas, em apenas uma delas (Lilja \& cols., 2003) se verificou a utilização de técnicas projetivas. Há, consequentemente, uma importante lacuna a ser preenchida nesse sentido. Afinal, as técnicas projetivas, por favorecerem a emissão de respostas que criam condições propícias para a exteriorização de conteúdos internos, possibilitam uma investigação detalhada da personalidade (Güntert, 2000) e, assim, são capazes de fornecer subsídios potencialmente proveitosos para o desenvolvimento de intervenções no campo da PsicoOncologia (Peres, Santos, Rodrigues \& Okino, 2007). Isso ocorre porque os estímulos veiculados invariavelmente são pouco estruturados, exigindo do examinado um intenso grau de criação e elaboração pessoal.

A exploração de fatores emocionais associados à recidiva do câncer de mama também se destaca como um tema promissor para novos estudos, dado que foi contemplado em apenas duas referências (Declerck \& cols., 2002; Okamura \& cols., 2005). Relações entre certas características de personalidade e indicadores prognósticos já foram esboçadas, mas diferenças em termos da estruturação e da dinâmica psíquica entre mulheres que conseguem recobrar a saúde e pacientes que são acometidas novamente pela enfermidade ainda são desconhecidas. A propósito, cumpre assinalar que a descoberta de uma recidiva é um evento dotado de grande poder disruptivo (Santos, Moscheta, Peres \& Rocha, 2005). São oportunas, portanto, tentativas de identificar preditores desse fenômeno. Finalmente, deve-se ressaltar a necessidade de desenvolvimento da produção científica nacional nessa área.

\section{Referências}

Abreu, E., \& Koifman, S. (2002). Fatores prognósticos no câncer de mama feminino. Revista Brasileira de Cancerologia, 48, 113-131.

Alves-Mazzotti, A. J., \& Gewandsnadjer, F. (2000). O método nas ciências naturais e sociais: pesquisa quantitativa e qualitativa. São Paulo: Pioneira.
Antoniazzi, A. S., Dell'Aglio, D. D., \& Bandeira, D. R. (1998). O conceito de coping: uma revisão teórica. Estudos de Psicologia, 3, 273-294.

Bleiker, E. M., Pouwer, F., Van der Ploeg, H. M., Leer, J. W., \& Ader, H. J. (2000). Psychological distress two years after diagnosis of breast cancer: Frequency and prediction. Patient Education and Counseling, 40, 209-217.

Carver, C. S., Meyer, B., \& Antoni, M. H. (2000). Responsiveness to threats and incentives, expectancy of recurrence, and distress and disengagement: Moderator effects in women with early stage breast cancer. Journal of Consulting and Clinical Psychology, 68, 965-975.

Carver, C. S., \& Scheier, M. F. (1994). Situational coping and coping dispositions in a stressful transaction. Journal of Personality and Social Psychology, 66, 184-195.

Carver, C. S., Smith, R. G., Antoni, M. H., Petronis, V. M., Weiss, S., \& Derhagopian, R. P. (2005). Optimistic personality and psychosocial well-being during treatment predict psychosocial well-being among long-term survivors of breast cancer. Health Psychology, 24, 508-516.

Cousson-Gelie, F. (2000). Breast cancer, coping and quality of life: A semi-prospective study. European Review of Applied Psychology, 50, 315-320.

Dalgalarrondo, P. (2000). Psicopatologia e semiologia dos transtornos mentais. Porto Alegre: ArtMed.

D’Andrea, F. F. (2000). Desenvolvimento da personalidade: enfoque psicodinâmico. Rio de Janeiro: Bertrandt.

Declerck, C. H., De Brabander, B., Boone, C., \& Gerits, P. (2002). Locus of control, marital status and predictors of early relapse in primary breast cancer patients. Psychology and Health, 17, 63-76.

Eysenck, H. J. (1990). Genetic and environmental contributions to individual differences: The three major dimensions of personality. Journal of Personality, 58, 245-261.

Fadiman, J., \& Frager, R. (1986). Teorias da personalidade (C. P. Sampaio \& S. Safolié, Trads.). São Paulo: Harper \& Row. (Trabalho original publicado em 1979)

Folkman, S., Lazarus, R. L., Dunkel-Schetter, C., DeLongis, A., \& Gruen, R. J. (1986). Dynamics of a stressful encounter: Cognitive appraisal, coping, and encounter outcomes. Journal of Personality and Social Psychology, 50, 992-1003.

Foratini, O. P. (1996). Epidemiologia geral. Porto Alegre: ArtMed.

Fredette, S. L. (1995). Breast cancer survivors: Concerns and coping. Cancer Nursing, 18, 35-46.

Gimenes, M. G. G. (1997). A teoria do enfrentamento e suas implicações para sucessos e insucessos em psiconcologia. Em M. G. G. Gimenes (Org.), A mulher e o câncer (pp. 111-147). Campinas: Psy.

Golden-Kreutz, D. M., \& Andersen, B. L. (2004). Depressive symptoms after breast cancer surgery: Relationships with global, cancer-related, and life event stress. Psycho-Oncology, 13, 211220.

Güntert, A. E. V. A. (2000). Técnicas projetivas: o geral e o singular em avaliação psicológica. Em F. F. Sisto, E. T. B. Sbardelini \& R. Primi (Orgs.), Contextos e questões da avaliação psicológica (pp. 77-84). São Paulo: Casa do Psicólogo.

Hartmann, H. (1958). Ego psychology and the problem of adaptation. New York: International Universities Press. 
Hogan, T. P. (2006). Introdução à prática de testes psicológicos (L. A. F. Pontes, Trad.). Rio de Janeiro: LTC. (Trabalho original publicado em 2003)

Holland, J. C. (1990). Historical overview. Em J. C. Holland \& J. Rowland (Orgs.), Handbook of psychooncology: Psychological care of the patient with cancer (pp. 3-12). New York: Oxford University Press.

Holland, J. C. (2002). History of psychooncology: Overcoming attitudinal and conceptual barriers. Psychosomatic Medicine, 64, 206-221.

Holland, J. C., \& Lewis, S. (2000). The human side of cancer: Living with hope, coping with uncertainty. New York: Harper Collins.

Instituto Nacional do Câncer. (2009). Estimativas 2010: incidência de câncer no Brasil. Rio de Janeiro: Ministério da Saúde.

Lazarus, R. S., \& Folkman, S. (1984). Stress, appraisal, and coping. New York: Springer.

Lazarus, R. S., \& Monat, A. (1984). Personalidade (V. Ribeiro, Trad.). Rio de Janeiro: Zahar. (Trabalho original publicado em 1979)

Lehto, U. S., Ojanen, M., \& Kellokumpu-Lehtinen, P. (2005). Predictors of quality of life in newly diagnosed melanoma and breast cancer patients. Annals of Oncolology, 16, 805-816.

Levin, T., \& Kissane, D. W. (2006). Psychooncology: The state of its development in 2006. European Journal of Psychiatry, 20, 189-197.

Lilja, A., Smith, G., Malmstrom, P., Salford, L. G., Idvall, I., \& Horstman, V. (2003). Psychological profile in patients with stages I and II breast cancer: Associations of psychological profile with tumor biological prognosticators. Psychological Reports, 92, 1187-1198.

Massie, M. J., \& Holland, J. C. (1991). Psychological reactions to breast cancer in the pre- and post- surgical treatment period. Seminars in Surgical Oncology, 7, 320-325.

Millar, K., Purushotham, A. D., McLatchie, E., George, W. D., \& Murray, G. D. (2005). A 1-year prospective study of individual variation in distress, and illness perceptions, after treatment for breast cancer. Journal of Psychosomatic Research, 58, 335-342.

Minayo, M. C. S., \& Sanches, O. (1993). Quantitativoqualitativo: oposição ou complementaridade? Cadernos de Saúde Pública, 9, 239-262.

Müller, M. C., Hoffmann, F. S., \& Fleck, P. (2006). A vivência do câncer de mama e a imagem corporal na mulher contemporânea. Em B. S. G. Werlang \& M. S. Oliveira (Orgs.), Temas em psicologia clínica (pp. 203-208). São Paulo: Casa do Psicólogo.

Okamura, M., Yamawaki, S., Akechi, T., Taniguchi, K., \& Uchitomi, Y. (2005). Psychiatric disorders following first breast cancer recurrence: Prevalence, associated factors and relationship to quality of life. Japanese Journal of Clinical Oncolology, 35, 302-309.

Peres, R. S., \& Santos, M. A. (2006). Relações entre a personalidade dos pacientes e a sobrevivência após o transplante de medula óssea: revisão da literatura. Psicologia em Estudo, 11, 341-349.

Peres, R. S., \& Santos, M. A. (2007). Breast cancer, poverty and mental health: Emotional response to the disease in women from popular classes. Revista Latino-americana de Enfermagem, 15, 786-791.
Peres, R. S.; Santos, M. A.; Rodrigues, A. M. \& Okino, E. T. K. (2007). Técnicas projetivas no contexto hospitalar: relato de uma experiência com o House-Tree-Person. Revista Iberoamericana de Diagnóstico y Evaluación Psicológica, 23, 41-62.

Piro, M., Zeldow, P. B., Knight, S. J., Mytko, J. J., \& Gradishar, W. J. (2001). The relationship between agentic and communal personality traits and psychosocial adjustment to breast cancer. Journal of Clinical Psychology in Medical Settings, 8, 263-271.

Ramos Júnior, J. (1991). Personalidade. São Paulo: Sarvier.

Rhodes, R. E., Courneya, K. S., \& Bobick, T. M. (2001). Personality and exercise participation across the breast cancer experience. Psycho-Oncology, 10, 380-388.

Rossi, L., \& Santos, M. A. (2003). Repercussões psicológicas do adoecimento e do tratamento em mulheres acometidas pelo câncer de mama. Psicologia: Ciência e Profissão, 23, 32-41.

Santos, M. A., Moscheta, M. S., Peres, R. S., \& Rocha, F. P. (2005). Atuação em psico-oncologia: atenção interdisciplinar a mulheres mastectomizadas. Em C. P. Simon, L. L. Melo-Silva \& M. A. Santos (Orgs.), Formação em Psicologia: desafios da diversidade na pesquisa e na prática profissional (pp. 379-394). São Paulo: Vetor.

Scheier, M. F., Carver, C. S., \& Bridges, M. W. (2001). Optimism, pessimism, and psychological well-being. Em E. C. Chang (Org.), Optimism and pessimism: Implications for theory, research and practice (pp. 189-216). Washington: American Psychological Association.

Schou, I., Ekeberg, O., Sandvik, L., Hjermstad, M. J., \& Ruland, C. M. (2005). Multiple predictors of health-related quality of life in early stage breast cancer: Data from a year follow-up study compared with the general population. Quality of Life Research, 14, 1813-1823.

Schultz, D. P., \& Schultz, S. E. (2002). Teorias da personalidade (E. Kanner, Trad.). São Paulo: Thomson. (Trabalho original publicado em 1994)

Schwartz, G. E., \& Weiss, S. M. (1978). Behavioral medicine revisited: An amended definition. Journal of Behavioral Medicine, 1, 249-251.

Seidl, E. M. F., \& Zannon, C. M. L. C. (2004). Qualidade de vida e saúde: aspectos conceituais e metodológicos. Cadernos de Saúde Pública, 20, 580-588.

Selye, H. (1965). The stress of life. McGraw-Hill: New York.

Sparremberger; F., Santos, I., \& Lima, R. C. (2003). Epidemiologia do distress psicológico: estudo transversal de base populacional. Revista de Saúde Pública, 37, 434-439.

Sugawara, Y., Akechi, T., Okuyama, T., Matsuoka, Y., Nakano, T., Inagaki, M., Imoto, S., Fujimori, M., Hosaka, T., \& Uchitomi, Y. (2005). Occurrence of fatigue and associated factors in diseasefree breast cancer patients without depression. Supportive Care in Cancer, 13, 628-636.

Tjemsland, L., Soreide, J. A., \& Malt, U. F. (1998). Posttraumatic distress symptoms in operable breast cancer III: Status one year after surgery. Breast Cancer Research and Treatment, 47, 141-151.

Tjemsland, L., Soreide, J. A., Matre, R., \& Malt, U. F. (1997). Properative psychological variables predict immunological status in patients with operable breast cancer. Psycho-Oncology, 6, 311320.

Urcuyo, K. R., Boyers, A. E., Carver, C. S., \& Antoni, M. H. (2005). Finding benefit in breast cancer: Relations with personality, coping, and concurrent well-being. Psychology and Health, 20, 175-192. 
Venâncio, J. L. (2004). Importância da atuação do psicólogo no tratamento de mulheres com câncer de mama. Revista Brasileira de Cancerologia, 50, 55-63.

Walker, L. G., Heys, S. D., Walker, M. B., Ogston, K., Miller, I. D., Hutcheon, A. W., Sarkar, T. K., Ah-See, A. K., \& Eremin, O. (1999). Psychological factors can predict the response to primary chemotherapy in patients with locally advanced breast cancer. European Journal of Cancer, 35, 1783-1788.

Wanderley, K. S. (1994). Aspectos psicológicos do câncer de mama. Em M. M. M. J. Carvalho (Org.), Introdução à psiconcologia (pp. 95-101). Campinas: Psy.
World Health Organization Quality of Life Group. (1994). The development of the World Health Organization Quality of Life assessment instrument (WHOQoL). Em J. Orley, \& W. Kuyken (Orgs.), Quality of life assessment: International perspectives (pp. 41-60). Heidelberg: Springer Verlag.

Recebido em 16.05.08 Primeira decisão editorial em 01.06.09 Versão final em 12.06.09 Aceito em 17.09.09 